\title{
Optical Aberrations and Objective Choice in Multicolor Confocal Microscopy
}

BioTechniques 28:542-550 (March 2000)

\author{
Kenneth W. Dunn and Exing \\ Wang \\ Indiana University School of \\ Medicine, Indianapolis, IN, \\ USA
}

\section{INTRODUCTION}

Over the past ten years, confocal microscopy has developed from a technique limited to specialists in microscopy into a standard research tool. Its proliferation of applications results as much from the rapid technological developments in confocal microscopy as from the maturing user interface of commercial confocal microscope systems. The latest systems are nearly turnkey systems with which even novice microscopists can rapidly collect high-quality images. Ironically, the same technical developments that have stimulated the spread of confocal microscopy in experimental biology have also pushed the limits of the optics of confocal microscopes in a way that understanding the optical properties of a confocal microscope is more important than ever.

The most common application of confocal microscopy is to compare the distributions or behaviors of multiple probes in the same cells. Such studies have been made possible by the development of confocal microscopes that are capable of efficiently collecting multiple colors of fluorescence and developing new dyes that have extended the useful spectrum of fluorescence microscopy (3). Depending on the configuration of the microscope, such studies may require optics that use wavelengths of light ranging from the ultraviolet through the infrared. The requirements for accurate color imaging have been further increased by the development of colorimetric methods of quantitative microscopy, such as fluorescence ratio measurements of ion concentrations.

Freedom from chromatic aberration is only part of the equation of optical design, which also considers monochro- matic aberrations and parameters such as high photon efficiency, field size, field flatness, working distance and the ability to image deep into aqueous biological tissues. Since the design of microscope optics reflects a compromise of these various parameters, manufacturers typically design a variety of different microscope objectives, with each representing a particular set of design trade-offs and each suited to a particular application. The studies presented here demonstrate that the choice of microscope objective can have profound effects on the results of confocal microscopy experiments. They emphasize the importance of carefully choosing a microscope objective that is appropriate for the experimental application.

\section{MATERIALS AND METHODS}

\section{Cell Lines and Culture}

MDCK strain II cells used in these studies were transfected with both the human transferrin receptor (Tf) and the rabbit polymeric immunoglobulin receptor. Cells were grown in MEM (Life Technologies, Gaithersburg, MD, USA) with $8 \%$ FBS, $1 \%$ L-glutamine, streptomycin and $0.05 \%$ hygromycin (Calbiochem-Novabiochem, San Diego, CA, USA). Cells were passed every 3 to 4 days and growth medium changed daily. New cultures of cells were thawed every 4 to 5 weeks.

\section{Fluorescent Ligands}

Tf was obtained from Sigma (St. Louis, MO, USA), iron-loaded and purified by $\$ 300$ column purification as described in Reference 20. With the 
exception of Cyanine 5.18 succinimidyl ester (Cy5) (Amersham Pharmacia Biotech, Piscataway, NJ, USA), all fluorescent probes and fluorescent beads were obtained from Molecular Probes (Eugene, OR, USA). All other reagents were obtained from Sigma. Fluorescent conjugates of Tf were prepared from succinimidyl esters of various fluorophores according to manufacturers' instructions.

\section{Labeling of Cells with Fluorescent Ligands}

For fluorescence labeling, cells were incubated with $20 \mu \mathrm{g} / \mathrm{mL}$ fluorescent Tf in Medium $1(150 \mathrm{mM} \mathrm{NaCl}, 20$ mM HEPES, $1 \mathrm{mM} \mathrm{CaCl}_{2}, 5 \mathrm{mM} \mathrm{KCl}$, $1 \mathrm{mM} \mathrm{MgCl} 2,10 \mathrm{mM}$ glucose, $\mathrm{pH}$ 7.4) for $20 \mathrm{~min}$ at $37^{\circ} \mathrm{C}$ on a slide warmer in a humidified chamber. After incubations with fluorescent ligands, filters were rinsed briefly in PBS at $4^{\circ} \mathrm{C}$, then incubated in $4 \%$ paraformaldehyde in a medium that contains $80 \mathrm{mM}$ PIPES, pH 6.5, $5 \mathrm{mM}$ EGTA and $2 \mathrm{mM} \mathrm{MgCl}_{2}$ for $5 \mathrm{~min}$ at $4^{\circ} \mathrm{C}$, then transferred to $4 \%$ paraformaldehyde in $100 \mathrm{mM} \mathrm{NaB}_{4} \mathrm{O}_{7}$ $\mathrm{pH} 11.0$, for $10 \mathrm{~min}$ at $4^{\circ} \mathrm{C}$. Filters were then rinsed in PBS.

\section{Microscopy}

All experiments were conducted using a Model MRC-1024 laser-scanning confocal attachment (Bio-Rad Laboratories, Hercules, CA, USA) mounted on a Diaphot 200 inverted microscope using a $60 \times$, N.A. 1.2 Plan Apochromat water immersion objective, a $40 \times$ N.A. 1.3 Plan Fluor oil immersion objective or a $100 \times$ Plan Apochromat DIC N.A. 1.4 oil immersion objective (all from Nikon Image Systems, Columbia, MD, USA). Illumination was provided by a Krypton-Argon laser (American Laser, Salt Lake City, UT, USA) providing for fluorescence excitations at 488,568 and $647 \mathrm{~nm}$ and allowing the collection of up to three images simultaneously. Image volumes were gathered by collecting a vertical series of images, with focus control provided by a precision microstepping motor. Photomultiplier offsets were set so that the background was slightly positive to guarantee signal linearity with fluorescence. Whenever possible, signal saturation was avoided, and objects with saturated pixels were omitted from quantifications. Reflection images were collected by illuminating with all laser lines and collecting images using a blue band-pass filter to select $488 \mathrm{~nm}$ reflection in channel 2 and a $585 \mathrm{~nm}$ long pass filter to select $647 \mathrm{~nm}$ reflection in channel 1. Detector apertures were set to 3.0 $\mathrm{mm}$ for all color endosome imaging and to approximately 1 Airy diameter for all other imaging.

\section{Image Processing}

Image processing was conducted using Metamorph ${ }^{\circledR}$ software (Universal Imaging, West Chester, PA, USA). Images shown in figures were contrast stretched to enhance the visibility of dim structures, and specific care was taken never to enhance the contrast so that dim objects were deleted from an image. Different focal planes from the same field were contrast enhanced identically, except where noted. Montages were assembled and annotated using Adobe ${ }^{\circledR}$ Photoshop ${ }^{\circledR}$.

Distinction of individual endosomes and quantification of endosome fluorescence were conducted as previously described (18). For endosome fluorescence ratio calculations, the common non-zero pixels for each endosome were determined, and the fluorescence ratio within that region was calculated. As much as possible, detector saturation was avoided during collection, but when pixel saturation occurred, objects with pixels within $10 \%$ of saturation (with a gray level of 230 or above in the original image) were removed from analysis. The methods used here are as described previously $(6-8,15)$. All ratio distributions were scaled to a mean of 1.0 for comparison.

\section{RESULTS}

An ideal lens would focus all colors of light to the same point. In reality, all lenses have chromatic aberration, a property in which different colors of light are focused to different points. When observing a sample through the microscope eyepiece, this defect makes objects appear to have colored edges. When imaging a sample in color confo- cal microscopy, this defect results in different colors of excitation illumination being focused to different points in the sample and different colors of emission being collected from different points in the sample. Horizontal displacements in the image plane, termed lateral chromatic aberration, result in different magnification of different colors. This problem can be minimized by restricting the analysis to the center of the microscope field. However, vertical color displacements along the focal axis, referred to as axial chromatic aberration, are present throughout the microscope field. This is clearly a problem for any researcher trying to use color confocal microscopy to determine the relative distribution of multiple probes. The images in Figure 1 show how the results of color confocal imaging critically depend on the nature of the microscope objective.

In Figure 1, we compared the performance of a Plan Fluor 40× objective, which is designed for maximum UV light transmission, with that of a Plan Apochromat $100 \times$ objective, designed with minimal chromatic aberration. We collected a vertical series of images of the reflection from the face of a glass coverslip, using either 488 or $647 \mathrm{~nm}$ of light. As the different colors of light are reflected by a single surface, a lens free of chromatic aberration would focus the different colors to the same focal plane. When reproduced as a vertical cross section, images collected with an ideal lens would show a single horizontal line in which the two colors overlap completely. The top half of Panel A demonstrates that the Plan Apochromat $100 \times$ objective does a credible job of approaching this ideal, resolving the two colors of light to a depth within 0.1 $\mu \mathrm{m}$ of one another. In contrast, the minimally corrected $40 \times$ plan Fluor detects the reflection of $647 \mu \mathrm{m}$ light approximately $1.2 \mu \mathrm{m}$ above the $488 \mathrm{~nm}$ light.

The effect of this vertical discrepancy is apparent in fluorescence. The bottom half of this panel shows vertical crosssections of image volumes of beads labeled with three fluorophores. When imaged with the 100× Plan Apochromat objective, the three colors are coincident, resulting in a white, reasonably round image. In contrast, the $40 \times$ objective generates an image in which the far- 
red fluorescence (depicted in blue) is distinctly vertically displaced from that of either the red or green fluorescence. In addition, a slight horizontal displacement of the far-red fluorescence reflects lateral chromatic aberration.

To provide a biological test specimen, we took advantage of the fact that endosomes can be labeled by incubating cells with fluorescently tagged endocytic ligands. The large number of molecules included in each endosome ensures that each will contain the same ratio of fluorescent probes. Thus, endosomes provide an excellent test of color confocal imaging as they present subresolution objects in biological samples with color that will reflect the relative fluorescent contributions of each fluorophore. Figure 1, Panel B shows a color image of cells in which its endosomes have been labeled with both fluoresceintransferrin (F-Tf, which fluoresces green) and Cy5-transferrin (Cy5-Tf, which fluoresces far-red) collected using the 100× Plan Apochromat objective. The co-localization of the two probes is apparent in the constant yellow-orange colors of the individual endosomes and is more apparent in comparisons of the high magnification images of F-Tf (Panel C) and Cy5-Tf (Panel D).

When switching to the $40 \times$ Plan Fluor objective, however, slight differences in the focal plane are apparent even between the red and green fluorescence, as shown in cells labeled with $\mathrm{Tf}$ conjugated to both fluorescein and rhodamine (Panel E). The differences in focal planes between far-red and green fluorescence result in startling differences in the apparent distributions of FTf and Cy5-Tf, which now appear to be completely distinct (Panel F).

For a cell biologist who is assessing the relative distributions of various probes, these displacements would have disastrous consequences. The discrepancy in focal plane can be circumvented by summing the entire vertical series of the image volume into a single projection, which removes the effect of differences in the focal plane of the two colors (Panel G). The consistent yellow color of every endosome in the projection demonstrates the constant ratio of the two probes in each endosome. However, as this procedure discards all vertical information, it is seldom an ap- propriate way of presenting confocal images. The effects of axial chromatic aberration can also be minimized by measuring the axial offset of different colors and combining the images collected from the focal planes appropriate for each color. An example of this procedure is shown in Panel $\mathrm{H}$, which shows the combined green and far-red images collected $1.2 \mu \mathrm{m}$ apart with the $40 \times$ Plan Fluor objective. The correction achieved by combining images from different focal planes is apparent when comparing the images of the individual probes in Panels I-K. Whereas Panels I and $\mathrm{J}$ show poor agreement in distribution between F-Tf and Cy5-Tf in images collected at the same focal plane, a comparison of Panels $\mathrm{J}$ and $\mathrm{K}$ shows that the far-red image can be superimposed with green fluorescence image collected $1.2 \mu \mathrm{m}$ deeper.

While chromatic aberration results in different colors of light being focused to different points in the image volume, spherical aberration can
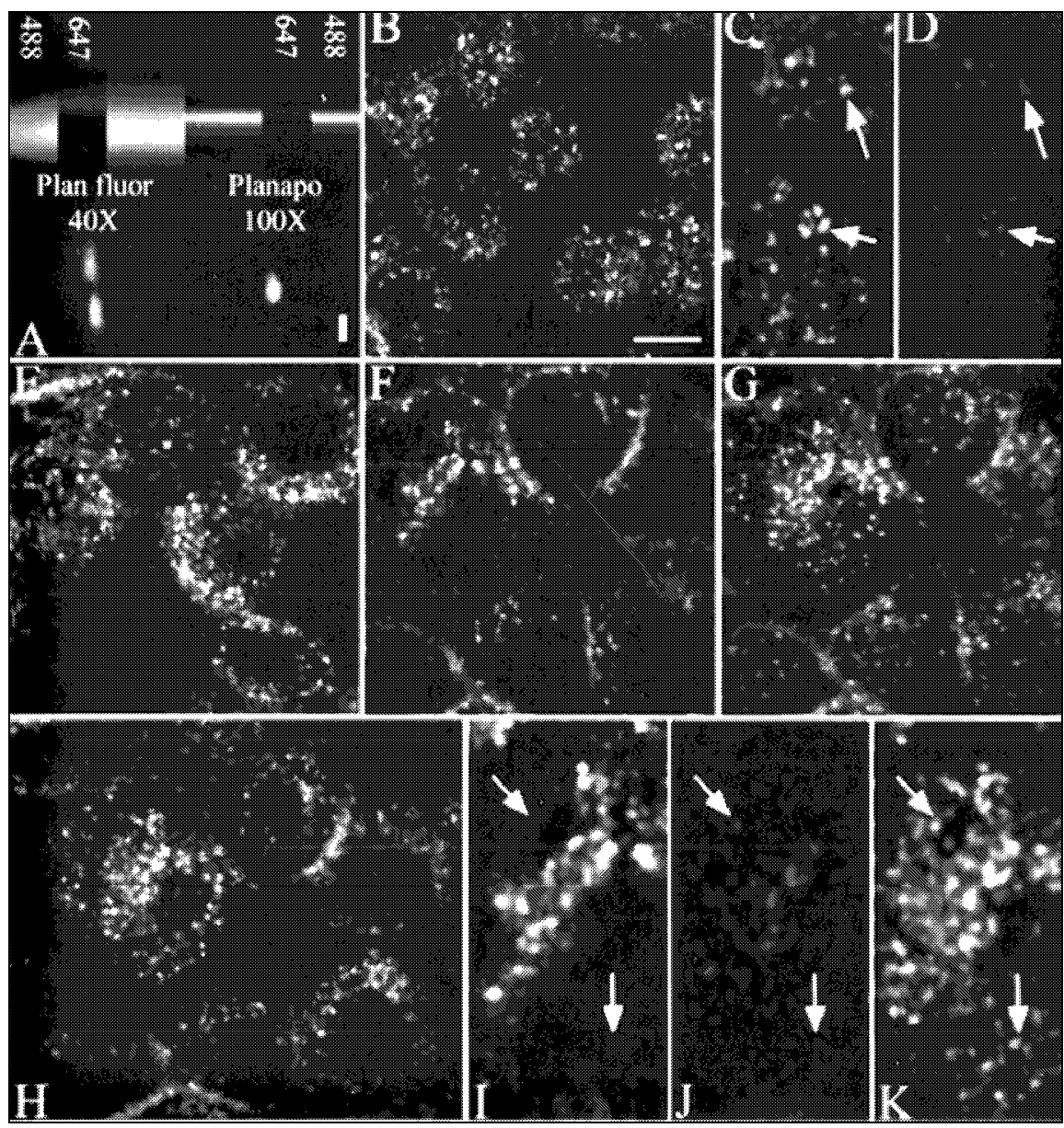

Figure 1. Chromatic aberration in confocal microscopy. A) XZ-sections of glass reflection (top) and microsphere fluorescence (bottom) images collected with a poorly color-corrected Plan Fluor $40 \times$ objective (left) and a well corrected Plan Apochromat 100× objective (right). In the reflection images at the top of this panel, $647 \mathrm{~nm}$ light is shown in red and $488 \mathrm{~nm}$ light is shown in blue. In the fluorescence images of fluorescent beads, $520 \mathrm{~nm}$ emissions are shown in green, $600 \mathrm{~nm}$ emissions are shown in red and $680 \mathrm{~nm}$ emissions are shown in blue. For all images, the focal axis is oriented vertically, with the scale bar indicating a distance of $1 \mu \mathrm{m}$. B) An image of a field of cells labeled with both F-Tf and Cy5-Tf collected with the $100 \times$ Plan Apochromat objective. Higher magnification images of the component green and far-red fluorescence are shown in Panels $C$ and D, respectively. E) An image of a field of cells labeled with F-R-Tf collected with the 40× Plan Fluor objective. F) An image of a field of cells labeled with both F-Tf and Cy5-Tf collected with the $40 \times$ Plan Fluor objective. G) The projection of the vertical series of images from which panel $\mathrm{F}$ was obtained. $\mathrm{H}$ ) The same field shown in $\mathrm{F}$, but combining green and far-red planes collected 1.2 $\mu \mathrm{m}$ apart. Higher magnification images of the green and far-red fluorescence from the same focal plane are shown in Panels I and J, while Panel K shows the green fluorescence from a focal plane $1.2 \mu \mathrm{m}$ lower. Scale bar in B represents a $10 \mu \mathrm{m}$ length for Panels B-H, $5 \mu \mathrm{m}$ in length for Panels I-K. 
profoundly decrease the signal in a confocal microscope. A spherically aberrated lens focuses axial and peripheral rays to different points, thus blurring the image of a point source of light. In the same way that the confocal pinhole so effectively improves image contrast by rejecting out-of-focus light, it effectively eliminates much of the fluorescence of an object imaged with spherical aberration (17).

For many samples, the primary source of spherical aberration derives from the difference between the refractive index of the immersion medium and the mounting medium $(11,16)$. Until recently, the highest-resolution, bestcorrected microscope objectives were designed for use with oil as an immersion fluid. For these objectives, spherical aberration is minimized only when the entire light path has the refractive index of immersion oil (which is the same as that of glass) and accumulates with distance into a medium with a different refractive index. Since most samples-particularly living samples-are mounted in media with a refractive index that is significantly lower than immersion oil, spherical aberration has

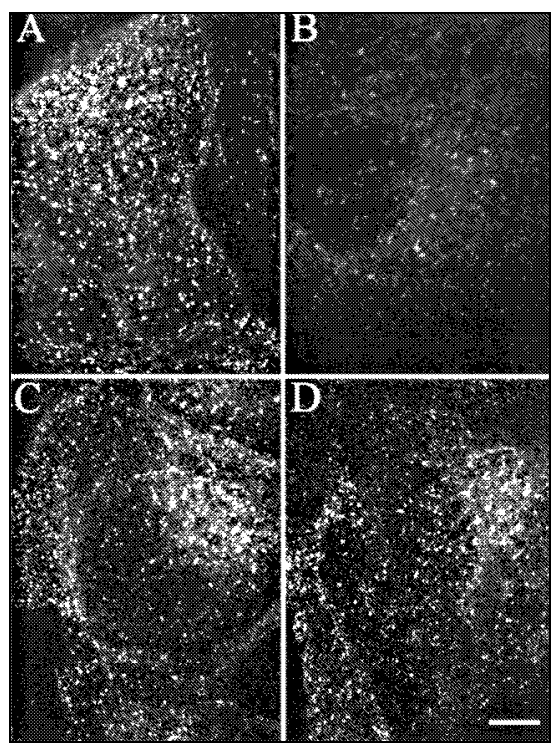

Figure 2. Spherical aberration in confocal microscopy. Images of cells labeled with F-Tf and imaged with either the $100 \times$ Plan Apochromat oil immersion objective (A and B) or the $60 \times$ Plan Apochromat water immersion objective ( $\mathrm{C}$ and D). Endosomes were imaged at the surface of the coverslip Panels A and C or at a depth of $35 \mu \mathrm{m}$ (Panel B) or $66 \mu \mathrm{m}$ (Panel D) into the aqueous sample medium. Scale bar is $10 \mu \mathrm{m}$ in length. thus limited the depth of image volumes using oil immersion objectives.

Figure 2 shows the effects of spherical aberration in confocal microscopy, where the oil immersion 100× Plan Apochromat objective was used to collect an image of cells labeled with F-Tf and mounted at a depth of either $0 \mu \mathrm{m}$ (Panel A) or $35 \mu \mathrm{m}$ into an aqueous medium (Panel B). In both cases, the endosomes appear sharply defined, but the accumulated spherical aberration of a light path $35 \mu \mathrm{m}$ into an aqueous medium has profoundly compromised the fluorescent signal in Panel B.

Recently, optical manufacturers have addressed this problem by designing objectives that use water as the objective immersion medium. For aqueous samples, the matched refractive index of immersion and sample media makes spherical aberration independent of imaging depth. This enables these objectives to collect images as deeply as the working distance permits, frequently hundreds of micrometers into a sample. The success of this design is shown in Figure 2, which shows images of cells labeled with F-Tf, collected either 0 (Panel C) or $66 \mu \mathrm{m}$ into an aqueous medium (Panel D) using a water immersion 60× Plan Apochromat objective. Here, the fluorescent signal is unaffected by the light path through the aqueous sample medium. This new generation of high numerical aperture, Plan Apochromat water immersion objectives have significantly helped confocal microscopy realize its potential in three-dimensional biological imaging.

The color performance of this water immersion objective falls between that of the oil immersion Plan Fluor and the Plan Apochromat objectives discussed previously. The cross-sectional images of the reflection of a glass surface in the top half of Figure 3A show that $647 \mathrm{~nm}$ of light are focused approximately 0.6 $\mu \mathrm{m}$ deeper than $488 \mathrm{~nm}$ light. Similar patterns for volumes collected either 0 or $63 \mu \mathrm{m}$ into an aqueous medium show that this discrepancy is independent of imaging depth.

The bottom-left image of Figure 2, Panel A shows that this color discrepancy results in the far-red image of a triple-labeled bead being displaced from that of either the red or green fluorescence images. The bottom-right por- tion of this panel demonstrates the critical importance of coverslip thickness correction in minimizing spherical aberration in this kind of objective. Since the correction for spherical aberration depends on the length of the optical path through the coverslip, it is adjustable by a collar that is set according to the thickness of the coverslip. While the bead cross section on the left was collected with the collar set to the measured coverslip thickness $(174 \mu \mathrm{m})$, the image on the right was collected with the collar misadjusted to $150 \mu \mathrm{m}$. The spherical aberration that results from an improper collar setting severely attenuates the fluorescence signal and compromises vertical resolution. Although this misadjustment was chosen to dramatize the point, we emphasize that such an error is easily encountered in practice because the actual thickness of coverslips can vary by more than $40 \mu \mathrm{m}$ around their nominal value. Proper collar setting can only be ensured through measurements of individual coverslips.

The images shown in Figure 2, Panels B and C, were collected at a depth of $63 \mu \mathrm{m}$ into an aqueous buffer. Although the images appear sharp, the axial chromatic aberration of this objective has the effect of making the distributions of F-Tf and Cy5-Tf appear discrete (Panel B). Nonetheless, the projection of the vertical series of images of these cells (Panel C) shows that the two probes equally label all endosomes. In agreement with the reflection images shown in Panel A, axial chromatic aberration is similarly apparent in images of endosomes collected at a depth of $0 \mu \mathrm{m}$. Little difference is observed in the focal plane of red and green fluorescence in images of endosomes labeled with $\mathrm{Tf}$ conjugated to both fluorescein and rhodamine (Panel D), but F-Tf and Cy5-Tf again appear to label discrete populations of endosomes (Panel E).

The distribution of the two probes can be better compared when allowing for the difference in focal plane and combining the Cy5-Tf image with a fluorescein image collected $0.6 \mu \mathrm{m}$ deeper. The co-localization of the two probes is now apparent in the constant yellow color of the endosomes shown in Panel F. Apparent differences in the distribution of the two probes shown in images 
collected at a single focal plane (Panels $\mathrm{G}$ and $\mathrm{H}$ ) disappear when the Cy5-Tf image is compared with the F-Tf image collected 0.6 $\mu \mathrm{m}$ deeper (Panel I).

The effects of chromatic aberration can also be quantified by measuring fluorescence ratios in images of endosomes labeled with multiple probes. Figure 4, Panel A, shows that the ratio of rhodamine to fluorescein (red to green) emissions is reasonably constant for all three objectives. In contrast, Panel B shows that while the $100 \times$ Plan Apochromat still shows minimal variation in the ratio of Cy5 to fluorescein (far-red to green), the water immersion 60× Plan Apochromat shows more variation, and the $40 \times$ Plan Fluor shows even more. The effects of chromatic aberration in the $60 \times$ Plan Apochromat and $40 \times$ Plan Fluor are more apparent when the distributions for a single focal plane are compared to those measured in the projections of the vertical image series for each objective (Panels $\mathrm{C}$ and $\mathrm{D}$, respectively). In each case, the narrow distribution of fluorescence ratios in the projected images reports the nearly constant ratio of the two probes in the endosomes, which is misrepresented in any single focal plane image.

\section{DISCUSSION}

The studies presented here demonstrate vividly how chromatic and spherical aberrations compromise the performance of confocal imaging. At the same time, they emphasize the critical importance of objective choice in confocal microscopy. Poor color correction will lead to erroneous interpretations of the relative distributions of multiple probes, one of the major applications of confocal microscopy. Our ratio quantifications also demonstrate how chromatic aberrations compromise microscope quantitation. Spherical aberration resulting from mismatched immersion and sample media worsens vertical resolution and can obliterate fluorescence detection altogether. Although particularly apparent in the high-contrast images provided by confocal microscopy, these errors also compromise images collected by conventional epifluorescence and transillumination techniques.

Chromatic aberration has been char- acterized primarily with respect to UV fluorophores. Our studies demonstrate significant axial chromatic aberration in the far-red as well, a range increasingly used by microscopists with the development of new far-red fluorescent probes and lasers capable of exciting them. Although our studies involve characterization of point sources, these samples readily display problems that would be manifest (but not necessarily obvious) with more extensive probes. Such probes include the cytosolic dyes that are frequently used in fluorescence ratio measurements of ionic concentrations.
For example, fluorescence of the cytosolic $\mathrm{pH}$ indicator $\mathrm{SNARF}^{\circledR}{ }_{-} 1$ is excited by $488 \mathrm{~nm}$ light, and $\mathrm{pH}$ is measured from the ratio of fluorescence at $580 \mathrm{~nm}$ to that at $640 \mathrm{~nm}$. For measurements of the cytosolic $\mathrm{pH}$ of thin cells, it is easy to imagine how the fluorescence ratio can be influenced by the position of the cell relative to the separate focal planes of the green excitation light, the red emissions and the far-red emissions. Although these errors will frequently simply add variation to measurements (doubling or tripling standard deviations in our quantifications),
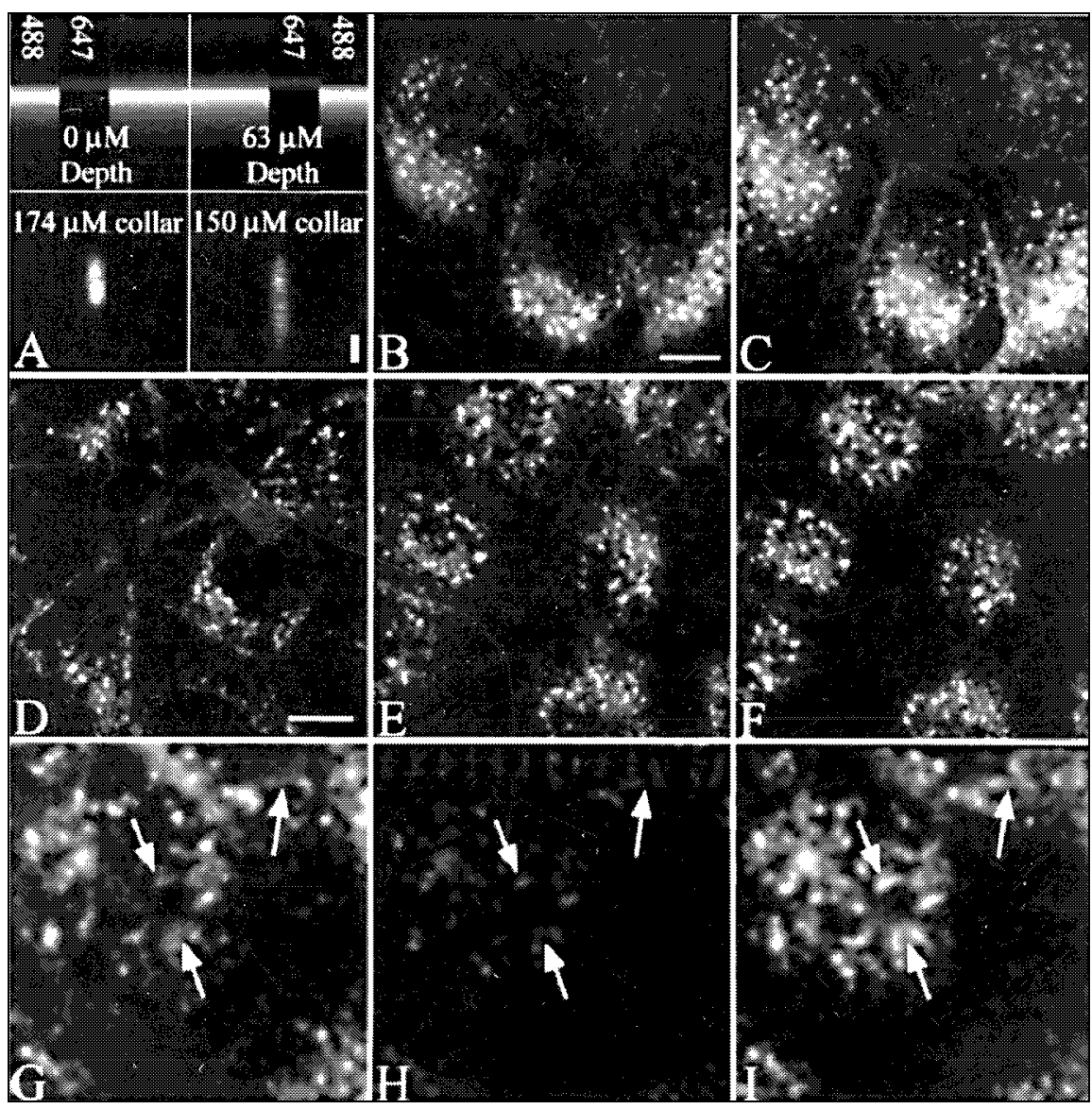

Figure 3. Chromatic aberration in the $60 \times$ Plan Apochromat water immersion objective. A) XZsections of glass reflection (top) and microsphere fluorescence (bottom) images. Reflection images are shown for depths of either 0 or $63 \mu \mathrm{m}$ into an aqueous medium, with $647 \mathrm{~nm}$ light shown in red and 488 $\mathrm{nm}$ light shown in blue. Fluorescence images of fluorescent beads are shown for an objective with correct (left) and incorrect (right) coverslip-thickness collar setting, with $520 \mathrm{~nm}$ emissions shown in green, 600 $\mathrm{nm}$ emissions shown in red and $680 \mathrm{~nm}$ emissions shown in blue. For all images, the focal axis is oriented vertically, with the scale bar indicating a distance of $1 \mu \mathrm{m}$. B) An image of a field of cells labeled with both F-Tf and Cy5-Tf collected at a depth of $63 \mu \mathrm{m}$ into an aqueous medium. C) The projection of the vertical series of images from which Panel B was obtained. D) An image of a field of cells labeled with F-R-Tf collected at the coverslip surface. E) An image of a field of cells labeled with both F-Tf and Cy5Tf collected at the coverslip surface. F) The same field shown in Panel E, but combining green and farred planes collected $0.6 \mu \mathrm{m}$ apart. Higher magnification images of the green and far-red fluorescence from the same focal plane are shown in Panel G and Panel H, while Panel I shows the green fluorescence from a focal plane $0.6 \mu \mathrm{m}$ lower. Scale bar in Panel B represents $10 \mu \mathrm{m}$ length in Panels B and C. The scale bar in Panel D represents $10 \mu \mathrm{m}$ length in Panels D-F and $5 \mu \mathrm{m}$ in Panels G-I. 
they can also systematically affect ratio measurements, for example, when comparing ratios in different parts of cells of varying thickness.

A final consequence of chromatic aberration, although not discussed here, is how it affects fluorescence detection (2). In a confocal system with chromatic aberration, the difference between the excitation and emission wavelengths results in the excitation of one volume and imaging of a different volume, attenuating the fluorescence signal. For beam-scanning systems (most confocal microscopes), this loss of signal worsens with distance off axis as the imaged spot is focused further and further from the confocal pinhole (19).

We emphasize that these observations are not unique to the particular objectives used in our studies or to a particular manufacturer. Our characterizations of the color correction were identically reproduced in three examples of the $60 \times$ water immersion objective and two examples of the $100 \times$ oil immersion objective on both Quantum and Diaphot microscope stands. Significant axial chromatic aberration has been identified in objectives from every major manufacturer $(1,5,9,13,16)$. Indeed, the ubiquity of chromatic aberration has led to the development of alternative microscope designs that address
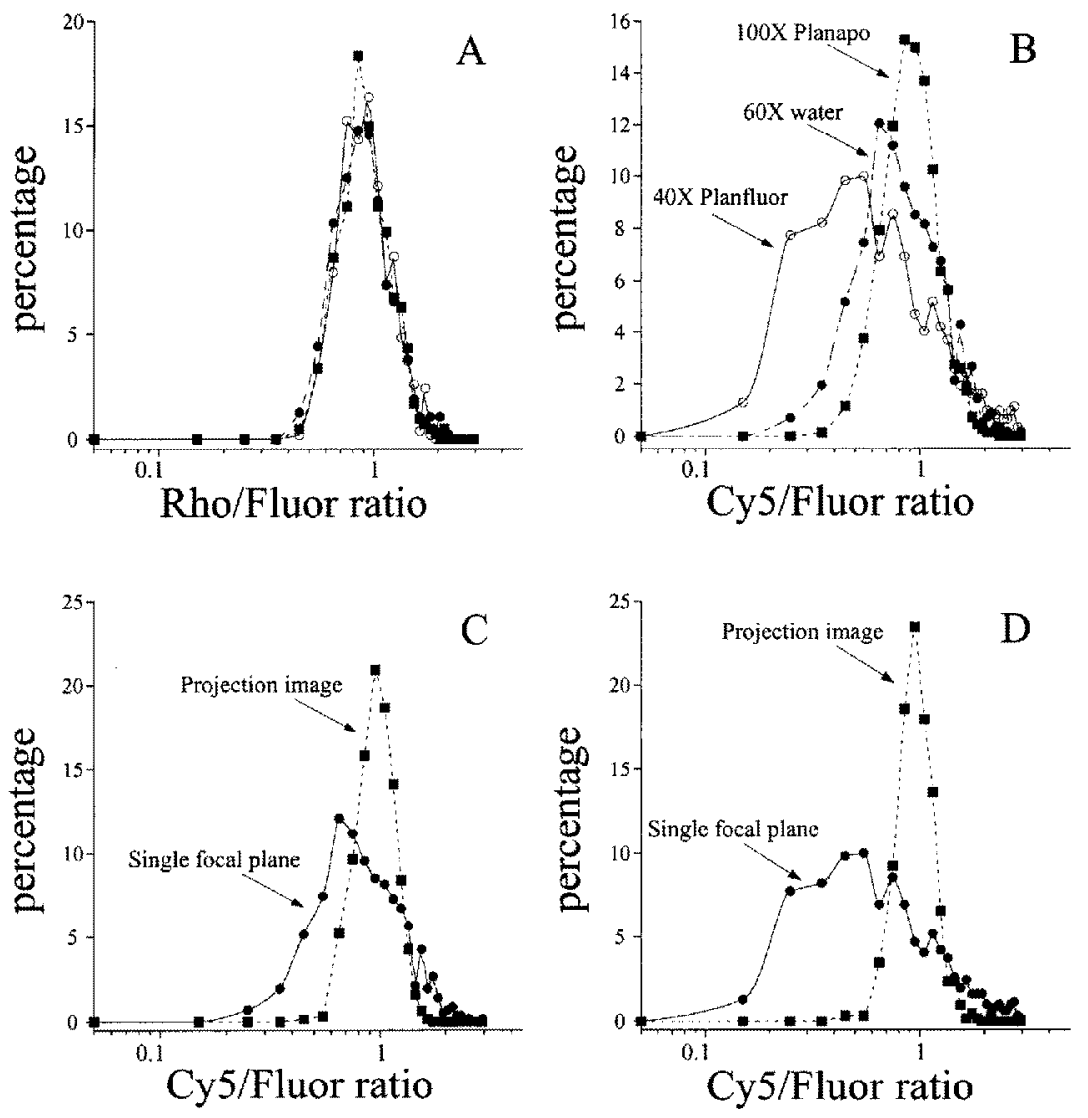

Figure 4. Effect of chromatic aberration on ratio quantification. A) Semi-logarithmic plots of ratio histograms for cells labeled with F-R-Tf, with images collected using the 100× Plan Apochromat (squares), the $40 \times$ Plan Fluor (open circles) and the $60 \times$ Plan Apochromat water immersion objective (closed circles). B) Ratio histograms of cells labeled with F-Tf and Cy5-Tf, with images collected using the $100 \times$ Plan Apochromat (squares), the $40 \times$ Plan Fluor (open circles) and the $60 \times$ Plan Apochromat water immersion objective (closed circles). C) Ratio histograms of cells labeled with F-Tf and Cy5-Tf, with images collected using the $60 \times$ Plan Apochromat water immersion objective. Circles show ratios of a single focal plane, while squares show ratios derived from projected images. D) Ratio histograms of cells labeled with F-Tf and Cy5-Tf, with images collected using the 40× Plan Fluor objective. Circles show ratios of a single focal plane, while squares show ratios derived from projected images. 
chromatic aberration either by imposing special auxiliary correcting lenses $(2,4)$ or avoiding refraction altogether and turning to reflecting objectives (1). Nonetheless, microscope optics is likely to continue to evolve, and preliminary experience with the new generation of CFI60 ${ }^{\mathrm{TM}}$ optics (Nikon, Inc.) indicates that axial chromatic correction is much improved.

Superficially, it may appear that some objectives are simply better than others. However, as discussed previously and described in more detail elsewhere $(14,16)$, objective design represents a compromise of design parameters. As such, each objective reflects a different set of design compromises according to its intended application. Nonetheless, it is possible that researchers will require more than what is currently available in optical design, forcing them to design experiments that do not stretch the limits of objective design.

If an oil immersion objective must be used to image a sample in an aqueous medium - for example, in studies of living cells-spherical aberration can be minimized either by minimizing the optical path through the aqueous medium or by using an oil with a refractive index tailored to the spherical aberration induced by the aqueous optical path $(10,12)$.

The problem of chromatic aberration can be minimized in several ways. If a particular experiment requires the use of an objective with significant chromatic aberration, the most obvious solution is to simply avoid the use of far-red fluorescing dyes such as $\mathrm{Cy} 5 \mathrm{al}-$ together. Our results demonstrate satisfactory agreement between green and red fluorescence with all the tested objectives. In general, it is best to use dyes with maximum excitation and emission near the specific wavelengths for which a particular objective has been corrected. A second solution, discussed above, is to collect images of each fluorophore in a vertical series of focal planes and combine the different color images according to the difference in focal plane between the two. This is a simple solution, but one that will not be appropriate when rapid image acquisition is necessary, as with live cells. Analyses not shown here also indicate that this solution does not have sufficient accuracy to correct images intended for ratio quantification. A more expensive (or less widely accessible) solution is to use two-photon microscopy, which is inherently unaffected by chromatic aberration as long as no detector pinhole is used. However, its multicolor capability is only now being explored.

\section{ACKNOWLEDGMENTS}

This work was supported by National Institutes of Health Grant No. R29DK51098 (to K.D.) and a fellowship from the American Heart Association, Indiana Affiliate, Inc. (to E.W.).

\section{REFERENCES}

1.Akinyemi, O., A. Boyde, M.A. Browne, M. Hadravsky and M. Petran. 1992. Chromatism and confocality in confocal microscopes. Scanning 14:136-143.

2.Bliton, A.C. and J.D. Lechleiter. 1995. Optical considerations at ultraviolet wavelengths in confocal microscopy. p. 431-444. In J.B. Pawley (Ed.), Handbook of Biological Microscopy. Plenum Press, New York.

3.Brelje, T.C., M.W. Wessendorf and R.L. Sorenson. 1993. Multicolor laser scanning confocal immunofluorescence microscopy: practical application and limitations. Methods Cell Biol. 38:97-181.

4.Cogswell, C.J., D.K. Hamilton and C.J.R. Sheppard. 1992. Colour confocal reflection microscope using red, green and blue lasers. J. Microsc. 165:103-117.

5.Cogswell, C.J. and K.G. Larkin. 1995 Imaging immunogold labels with confocal microscopy. p. 507-513. In J.B. Pawley (Ed.), Handbook of Biological Confocal Microscopy. Plenum Press, New York.

6.Dunn, K.W. and F.R. Maxfield. 1998. Ratio imaging instrumentation. Methods Cell Biol. 56:217-236.

7.Dunn, K.W., S. Mayor, J.N. Myers and F.R. Maxfield. 1994. Applications of ratio fluorescence microscopy in the study of cell physiology. FASEB J. 8:573-582.

8.Dunn, K.W., J. Park, C.E. Semrad, D.L. Gelman, T. Shevell and T.E. McGraw. 1994. Regulation of endocytic trafficking and acidification are independent of the cystic fibrosis transmembrane regulator. J. Biol. Chem. 269:5336-5345.

9.Fricke, M.D. and N.S. White. 1992. Wavelength considerations in confocal microscopy of botanical specimens. J. Microsc. 166:2942.

10.Gibson, S.F. and F. Lanni. 1992. Experimental test of an analytical model of aberration in an oil-immersion objective lens used in threedimensional light microscopy [corrected and republished article originally printed in J. Opt. Soc. Am. A. 1991 8:1601-13]. J. Opt. Soc.
Am. A Opt. Image Sci. Vis. 9:154-166

11.Hell, S.W. and E.H.K. Stelzer. 1995. Lens aberration in confocal fluorescence microscopy. p. 347-354. In J.B. Pawley (Ed.), Handbook of Biological Confocal Microscopy. Plenum Press, New York.

12.Hiraoka, Y., J.W. Sedat and D.A. Agard. 1990. Determination of three-dimensional imaging properties of a light microscope system. Partial confocal behavior in epifluorescence microscopy. Biophys. J. 57:325-333.

13.Hiraoka, Y., J.R. Swedlow, M.R. Paddy, D.A. Agard and J.W. Sedat. 1991. Three-dimensional multiple-wavelength fluorescence microscopy for the structural analysis of biological phenomena. Semin. Cell Biol. 2:153165.

14.Inoue, S. and K.R. Spring. 1997. Video Microscopy. Plenum Press, New York.

15.Johnson, L.S., K.W. Dunn, B. Pytowski and T.E. McGraw. 1993. Endosome acidification and receptor trafficking: bafilomycin A1 slows receptor externalization by a mechanism involving the receptor's internalization motif. Mol. Biol. Cell 4:1251-1266.

16.Keller, H.E. 1995. Objective lenses for confocal microscopy. p. 111-126. In J.B. Pawley (Ed.), Handbook of Biological Microscopy. Plenum Press, New York,

17.Majlof, L. and P.-O. Forsgren. 1993. Confocal microscopy: important considerations for accurate imaging. Meth. Cell Biol. 38:79-95.

18.Maxfield, F.R. and K.W. Dunn. 1990. Studies of endocytosis using image intensification fluorescence microscopy and digital image analysis. p. 357-371. In B. Herman and K. Jacobsen (Eds.), Optical Microscopy for Biology. Alan R. Liss, New York.

19.Sandison, D.R., R.M. Williams, K.S. Wells, J. Strickler and W.W. Webb. 1995. Quantitive fluorescence confocal laser scanning microscopy (CLSM). p. 39-53. In J.B. Pawley (Ed.), Handbook of Biological Microscopy. Plenum Press, New York.

20.Yamashiro, D.J., B. Tycko, S.R. Fluss and F.R. Maxfield. 1984. Segregation of transferrin to a mildly acidic ( $\mathrm{pH}$ 6.5) para-Golgi compartment in the recycling pathway. Cell 37:789-800.

Received 1 July 1999; accepted 1 November 1999.

\author{
Address correspondence to: \\ Dr. Kenneth Dunn \\ Department of Medicine \\ Indiana University \\ School of Medicine \\ 1120 South Drive, FH115 \\ Indianapolis, IN, 46202-5116, USA \\ Internet:kdunn@iupui.edu
}

